\title{
FH - HES
}

\section{Stereoselective Bioreduction to a Chiral Building Block on a Kilogram Scale}

\author{
Christelle Jablonski-Lorin $^{a \star}$, Vito Melillob ${ }^{\mathrm{b}}$, and Ernst Hungerbühler ${ }^{\mathrm{a}}$
}

\begin{abstract}
The highly stereoselective bioreduction of ethyl cyclohexanone-2-carboxylate (1) on a kilogram scale is described. Ethyl(1S,2S)-trans-2-hydroxycyclohexane carboxylate (2) was prepared in 56\% yield with a diastereomeric ratio (dr) of 99:1 and an enantiomeric ratio (er) of 99.5:0.5 after rectification. The cell growth and the biotransformation were performed in a large wave bioreactor equipped with a 200 I cell bag at Novartis Pharma AG, while the different purification steps were performed at the FHBB (Muttenz). The fourth semester chemistry students at the FHBB were actively involved in the preparation and optimization of the entire process.
\end{abstract}

Keywords: Biotransformation $\cdot$ High stereoselectivity $\cdot$ Scale-up process $\cdot$ S. pombe yeast $\cdot$ Wavebioreactor

\section{Background}

Interest in asymmetric synthesis has increased very rapidly during the last few years due to the pharmaceutical, vitamin and agro industries' need to produce enantiomerically pure compounds rather than racemates.

In 1990, nearly $90 \%$ of all chiral synthetic drugs sold were racemic [1]. The awareness of the differences in pharmacological properties of a chiral compound with its enantiomer and its racemate increased and the necessity to consider two enantiomers as two different compounds when screened as drug candidates emerged.

\footnotetext{
${ }^{\star}$ Correspondence: Prof. Dr. E. Hungerbühler Tel.: +41614674388

Fax: +41614674457

E-Mail: e.hungerbuehler@fhbb.ch

c.jablonski@fhbb.ch

aFachhochschule beider Basel (FHBB)

Gründenstrasse 40

$\mathrm{CH}-4132$ Muttenz

bSynphaBase AG

Gründenstrasse 40

$\mathrm{CH}-4132$ Muttenz
}

Among the different technologies so far developed (resolution of racemic mixtures, use of natural products as chiral source, use of chiral reagents or catalysts, etc.), biotransformations involving enzymes or microorganisms have been shown to be an interesting alternative method offering highly stereoselective reactions with the diastereomeric ratio (dr) and enantiomeric ratio (er) often higher than 98:2, under mild and cheap reaction conditions, as well as with environmentally safe procedures (reactions take place in water). However, the use of biotransformations on a large scale for the production of chiral building blocks remains limited due to the high dilution required and the laborious work-up procedures.

\section{Project Description}

A considerable internal know-how on yeast biocatalyzed reactions has been developed over the last ten years at the chemistry department of the University of Applied Sciences, Basel (FHBB) in the group led by Prof. Dr. E. Hungerbühler [2-4].

In connection with our ongoing project of asymmetric reduction of $\beta$-keto-esters [5], we investigated a scale-up with a factor
10 of the biotransformation described in the Scheme for the synthesis of kilogram amounts of hydroxyester 2. Ethyl(1S,2S)trans-2-hydroxycyclohexane carboxylate (2) is a versatile chiral building block and an important intermediate in pharmaceutical drug synthesis obtained by stereoselective reduction of the keto function of ethyl cyclohexanone-2-carboxylate (1) with an African beer yeast (Schizosaccharomyces pombe).

The project was carried out not only for educational purposes but also for commercial purposes in collaboration with our spin-off partner SynphaBase AG (www.synphabase.com).

The chemical procedure had previously been established and optimized on a small one-liter scale in the wave bioreactor for sterile cell culture by a group of students during a semester project (17 working days). The results were later confirmed at the FHBB on a volume of eight liters within a 20-1 bag on the same reactor.

Using the facilities of the fermentation group of Dr. K. Memmert at Novartis Pharma AG, the reaction was successfully performed on a 80-1 scale. A very good linearity could be observed on the three different scales (1 1, 81, and 801$)$. 


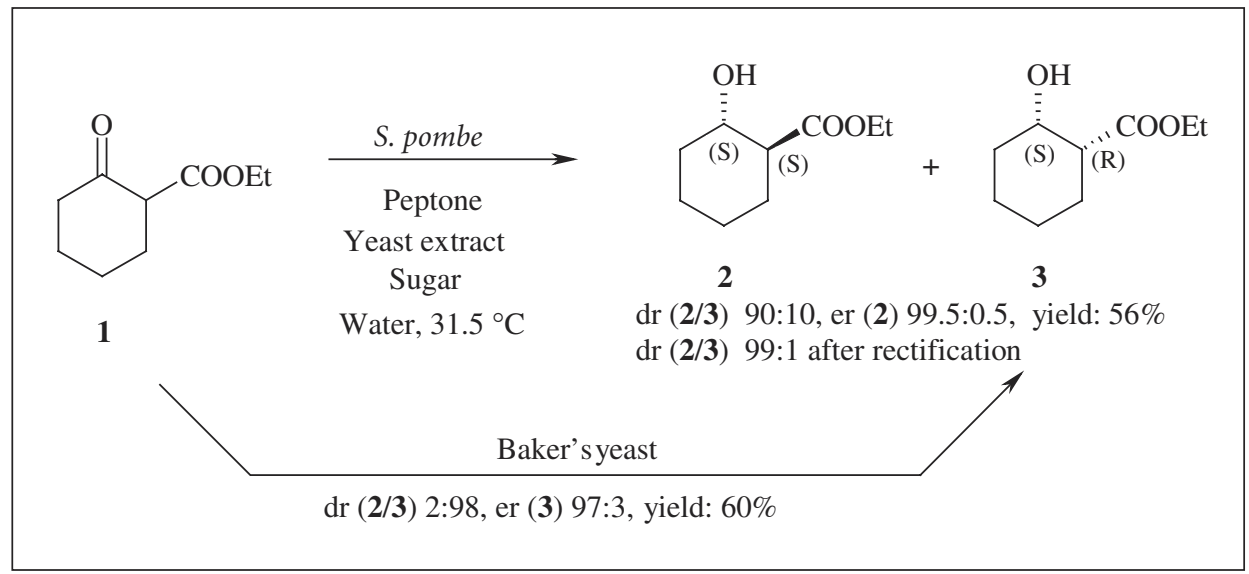

Scheme. Stereoselective bioreduction of the ethyl cyclohexanone-2-carboxylate (1) with S. pombe

The major difficulties of the scale-up were related to:

- the absolute sterile conditions required during the growth phase of the $S$. pombe cells and

- a different geometry of the 2001 cell bag compared to that of the 21 and 201 bags resulting in a change of the wave style and the set-up parameters. The oxygen ratio used during the scale-up biotransformation was $40 \%$ (air enriched in oxygen) compared to $20 \%$ during the previous experiments.

\section{Procedure}

601 of media made from sugar, peptone and yeast extract and 201 of a concentrate of sugar and calcium carbonate were sterilized $\left(121{ }^{\circ} \mathrm{C}\right.$ for $\left.20 \mathrm{~min}\right)$ in a $100 \mathrm{l}$ fermenter (Fig. 1).

A 21 sterile preculture of $S$. pombe cells was mixed with 51 of media in the $2001 \mathrm{bag}$ in the wave bioreactor (Fig. 2) and gradually grew to 601 of media.

Crucial parameters such as $\mathrm{pH}$ control, glucose concentration, and $\mathrm{O}_{2} / \mathrm{CO}_{2}$ concentration were checked every hour. The complete phase of cell growth was performed without contamination, the quality of the $S$. pombe culture checked on the microscope, the growth rate evaluated by counting cell numbers on the microscope and by measurement of the optical density.

After addition of the concentrate of sugar and calcium carbonate, $960 \mathrm{ml}$ of compound 1 were added dropwise. The biotransformation was achieved over $44 \mathrm{~h}$. The reaction mixture was saturated with sodium chloride, the work-up procedure (Fig. 3) afforded the separation of the product from the biomass. The crude material was then purified by solvent extractions. The separation of the diastereoisomers cis/trans was achieved by rectification.

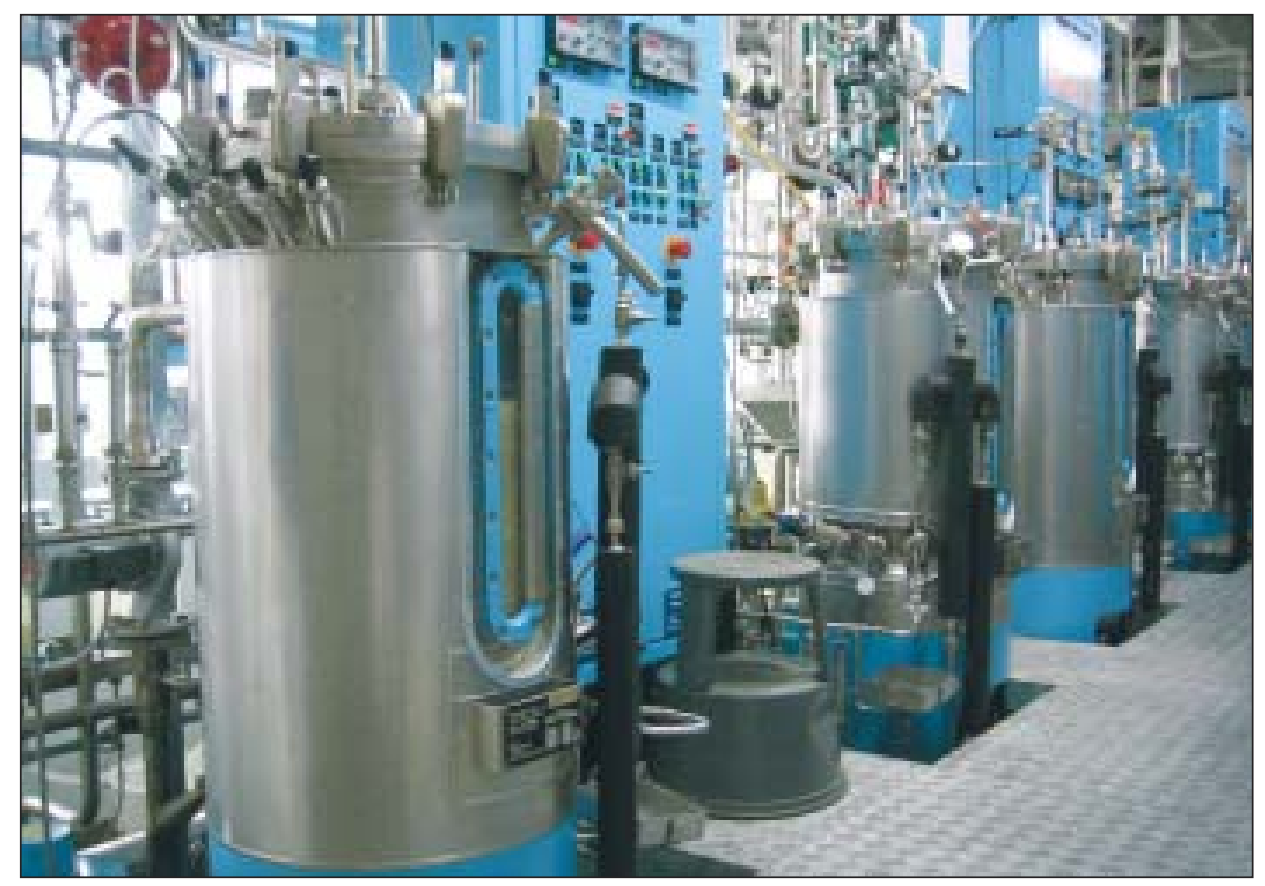

Fig. 1. Fermentor of $100 \mathrm{I}$ used for the media preparation (Novartis AG)

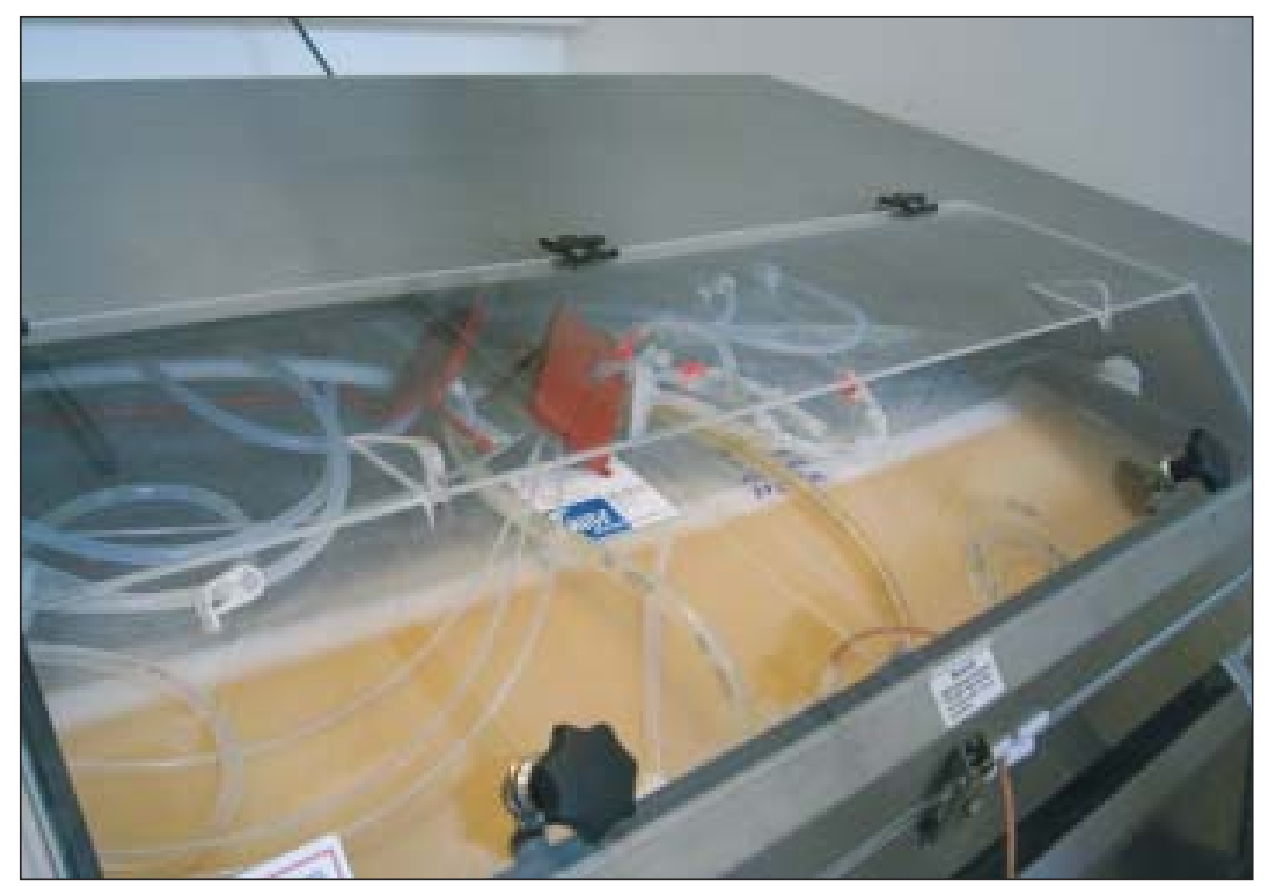

Fig. 2. Wavebioreactor and cell bag of 200 I (Novartis AG) 


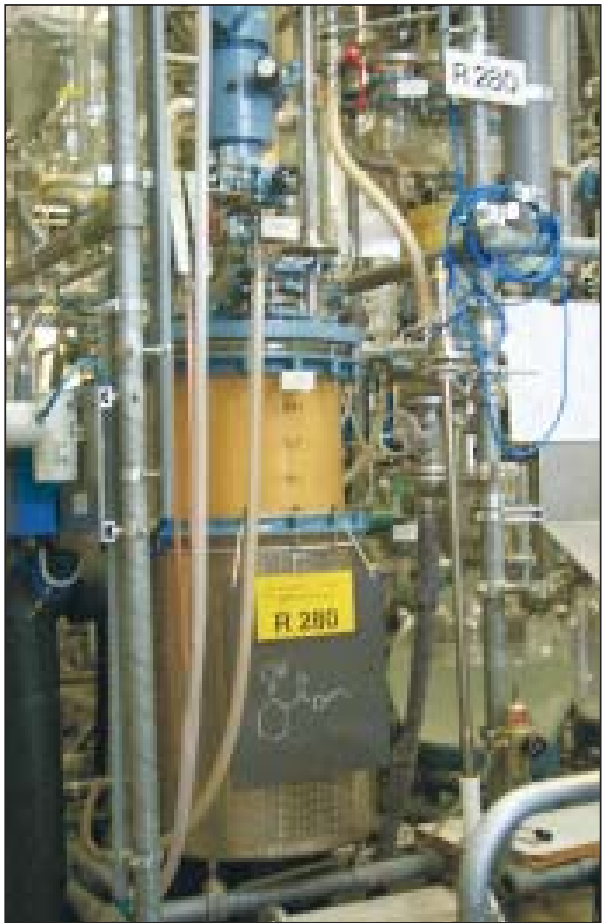

Fig 3. Reaction work-up (Chemistry Department FHBB)

\section{Results}

The results obtained for the scale-up at Novartis were similar to the results obtained on small scale at the FHBB.

$668 \mathrm{~g}$ of a mixture of trans/cis-2-hydroxycyclohexane ethylester $(\mathbf{2}, \mathbf{3})$ was synthesized with a ratio of 90:10 and purified over a limited period of time. The crude product was prepared in $64 \%$ yield.

The scale-up reaction finally enabled us to obtain after distillation the pure product 2 in $56 \%$ yield with a diastereomeric ratio (trans/cis) of $99: 1$ and a enantiomeric ratio of 99.5:0.5.

It is worth noting that, in contrast to the classical process, the biotransformation could be achieved without addition of antifoaming agent, which highly simplified the purification steps.

\section{Perspectives}

It was shown that the process developed on a small scale could be transferred to a large-scale production of an enantiomerically pure product without significant difficulties.

Further large scale biotransformations requiring the same know-how might be investigated in the future.

For more information please contact the authors (e.hungerbuehler@fhbb.ch or c.jablonski@fhbb.ch) and www.fhbb.ch

\section{Acknowledgements}

We would like to thank Dr. K. Memmert, M. Henke, and E. Weber for the use of the facilities at Novartis and for their help. We would like to thank H. Briellman for his technical assistance during the work-up process of the scale-up reaction in the Verfahrenstechnik Zentrum at the FHBB. We also thank Prof. Dr. C. de Virgilio for providing the strain of $S$. pombe and for his suggestion regarding the selection of the yeast. And finally thanks to G. Aspin, our Professor for English at the FHBB, for his help with the language.

[1] R.E. Gawley, J. Aubé, in 'Principles of Asymmetric Synthesis', Tetrahedron Organic Chemistry Series, Vol. 14, Ed. Pergamon, 1996.

[2] M. Bertau, M. Bürli, E. Hungerbühler, Chimica Oggi 1998, 58-61.

[3] M. Bertau, M. Bürli, E. Hungerbühler, Bioworld 1999, 1, 7-10.

[4] M. Bertau, M. Bürli, E. Hungerbühler, P. Wagner, Tetrahedron Asymmetr, 2001, 12, 2103-2107.

[5] The reduction of $\beta$-ketoesters with the Noyori catalyst led also to the corresponding hydroxyester with a high stereoselectivity - e.g. the ethyl $(1 S, 2 S)$-trans-2-hydroxycyclopentane carboxylate was synthesized with a dr and an er higher than 99:1. 